\title{
The assessment of atrial function by velocity-encoded magnetic resonance imaging
}

\author{
Charles C. Vu ${ }^{1}$, John F. Heitner ${ }^{2 *}$, Igor Klem ${ }^{3}$, Peter J. Cawley ${ }^{4}$, Anna Lisa C. Crowley ${ }^{3}$,
} Manesh R. Patel ${ }^{3}$, Jonathan W. Weinsaft ${ }^{5}$, Michele A. Parker ${ }^{3}$, Michael Elliott ${ }^{3}$, Robert M. Judd ${ }^{3}$, Raymond J. Kim ${ }^{3}$, Joseph C. Greenfield Jr. ${ }^{3}$

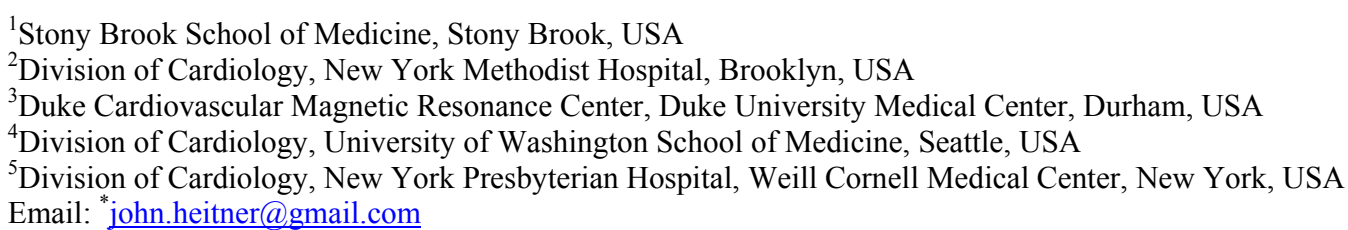

Received 14 January 2013; revised 13 March 2013; accepted 14 April 2013

Copyright (C) 2013 Charles C. Vu et al. This is an open access article distributed under the Creative Commons Attribution License, which permits unrestricted use, distribution, and reproduction in any medium, provided the original work is properly cited.

\begin{abstract}
Introduction: The purpose of this study was to assess velocity-encoded cardiac magnetic resonance imaging (Ve-CMR) in a population of patients referred for cardiac magnetic resonance imaging (CMR), to determine the variability of atrial function, and to identify clinical parameters associated with left atrial function. Methods: This is a prospective study evaluating patients who were referred to our CMR center for a clinical CMR. Left atrial function was obtained via Ve-CMR thru-plane images across the mitral valve after acquiring 2 perpendicular in-plane images as "scouts". The atrial function and mitral inflow were quantified by computer analysis (Argus, Siemens). Atrial function was defined as atrial contraction (Awave) volume divided by total inflow volume. Left atrial volumes were calculated via computer analysis. Mitral regurgitation and left ventricular ejection fractions were assessed visually. Results: Thirty-nine patients, with mean age $56+/-10$ years, were enrolled. The mean left atrial function was $22.9 \%+-14.5 \%$; the range in left atrial function was $0 \%-57 \%$. There was a significant positive correlation between atrial function and increased left ventricular ejection fraction $(r=0.44, P<0.01)$. There was a significant negative correlation between atrial function and severity of mitral regurgitation $(r=-0.60, P<0.01)$, as well as left atrial volume $(r=-0.36, P=0.02)$. Conclusion: Our results indicate a wide variability in left atrial function and a significant association between left atrial function and left ventricular ejection fraction,
\end{abstract}

${ }^{*}$ Corresponding author. left atrial volume and mitral regurgitation.

Keywords: Atrial Function; Velocity-Encoded; Cardiovascular Magnetic Resonance

\section{INTRODUCTION}

Atrial function is an important parameter in cardiac hemodynamics contributing significantly to left ventricular (LV) function [1]. Combined two-dimensional and Doppler echocardiography is the most widely used modality to assess the contribution of atrial systole to ventricular filling; two other less utilized modalities include ultrafast computed tomography and radionuclide angiography [25].

Cardiac magnetic resonance imaging (CMR) is a relatively new and emerging imaging modality that can be used to assess left atrial function. Early CMR methods have used volume assessments via cine-CMR for the assessment of left atrial function [6]. Velocity-encoded magnetic resonance imaging (Ve-CMR) is a technique that can measure both the velocity and the quantity of blood flow through the mitral valve during diastole, and thus can be used to quantify left atrial function. Due to its excellent spatial resolution, Ve-CMR can measure the passive early diastolic flow and the contribution of atrial systole to both left and right ventricular diastolic filling in normal subjects [7-10].

Previous studies using Ve-CMR have assessed left atrial function in small populations with either valvular stenosis or normal hearts $[8,9]$. This study is designed to assess this technique in a population of patients with a variety of indications for CMR, to determine the vari- 
ability of atrial function within this population, and to assess the clinical variables associated with left atrial function.

\section{METHODS}

\subsection{Ethics Statement}

At the time of the CMR study, written consent was obtained from all study participants and the institutional review board of Duke University Medical Center approved the study protocol. This study was in compliance with Health Insurance Portability and Accountability Act (HIPAA) guidelines.

\subsection{Patients}

The study group consisted of 39 prospectively enrolled patients. All patients were referred for a CMR study for the evaluation of myocardial viability, left ventricular systolic function assessment, or for an abnormal echocardiogram. Exclusion criteria were contraindications to CMR (e.g. size greater than the magnet bore, pregnancy, vascular clips, pacemakers, defibrillators, claustrophobia). All patients had a comprehensive history obtained at the time of their CMR study.

\subsection{CMR}

All scans were performed on a 1.5-Tesla scanner with a phased-array receiver coil (Siemens Sonata). Long- and short-axis scout images were used to determine the double-oblique long- and short-axis views.

Steady-state free-precession cine images were taken in three long-axis views, as well as in short-axis views of the entire left ventricle (slice thickness - $6 \mathrm{~mm}$; gap between slices $-4 \mathrm{~mm}$ ). Typical parameters used were: repetition time $3.0 \mathrm{~ms}$; echo time $1.5 \mathrm{~ms}$; flip angle $25^{\circ}$; temporal resolution $35 \mathrm{~ms} /$ phase; in-plane resolution 1.7 $\times 1.4 \mathrm{~mm}$.

A velocity-encoded gradient echo sequence was used to acquire in-plane phase-contrast and magnitude images in 2-chamber and 4-chamber long-axis views at the mitral annulus. Using these in-plane images as "scout images", through-plane images across the mitral valve were then taken (Figure 1). Typical parameters were: FOV $320 \mathrm{~mm}$; slice thickness $6 \mathrm{~mm}$; flip angle $25^{\circ}$; in-plane resolution $2.1 \times 1.4 \mathrm{~mm}$; temporal resolution $60 \mathrm{~ms} /$ phase interpolated to $30 \mathrm{~ms} /$ phase; echo time $3.0 \mathrm{~ms}$. Retrospective electrocardiogram gating was used. The typical VENC was between 80 and $120 \mathrm{~cm} /$ second.

Flow analysis was performed on the through-plane VeCMR phase-contrast and magnitude images acquired across the mitral valve. To determine blood flow and volume, region of interest (ROI) curves on the Ve-CMR phase-contrast images were drawn, with subsequent im-

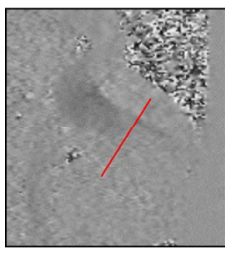

(a)

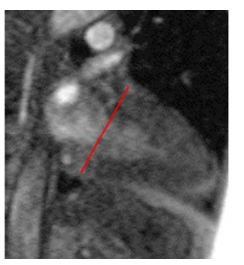

(b)

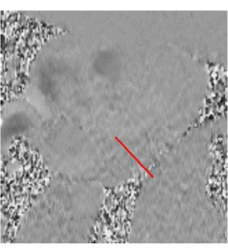

(c)

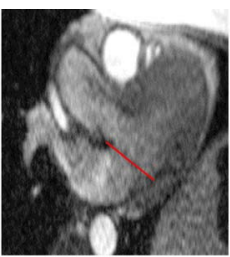

(d)

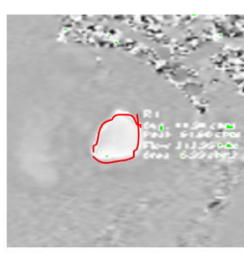

(e)

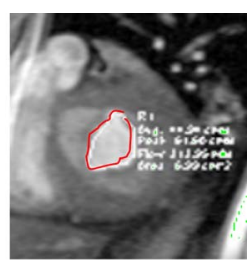

(f)
Figure 1. Representative example of Ve-CMR cine acquisition. In-plane phase contrast images were obtained in 2-chamber (a) and 3-chamber (c) cine long-axis views. Magnitude images were also obtained in the 2-chamber (b) and 3-chamber (d) cine longaxis views. Using these in-plane images, through-plane phase contrast (e) and magnitude (f) images were acquired across the mitral valve.

age analysis by commercial software (Argus, Siemens).

\subsection{Left Atrial Function}

Using the flow-time curve, the beginning and ending times of the E-wave (passive ventricular filling) and Awave (atrial contraction) were determined. The inflow volumes from passive ventricular filling and atrial contraction were calculated from the corresponding net flowtime curves (Figure 2). Left atrial function was defined as the atrial contraction volume divided by the total inflow volume.

\subsection{Other Cardiac Parameters}

Left ventricular wall thickness (anterior and posterior), end-diastolic diameter, and end-systolic diameter were measured from the mid-chamber cine short-axis view. Image analysis was done using commercial software (Argus, Siemens), with left ventricular ejection fraction being visually estimated by an experienced physician. PR interval and RR interval were calculated from the electrocardiogram.

Because quantitative evaluation of mitral regurgitation is not reliable, for each patient, the degree of mitral regurgitation was evaluated qualitatively from the cine MR images [11]. A mitral regurgitation score was assigned for each patient according to the following table: None $=$ 0 , Trivial $=1$, Mild $=2$, Moderate $=3$, Severe $=4$.

Lengths and widths of the left atrium were measured in the two-chamber and four-chamber cine views (equivalent to the apical two-chamber and apical long-axis imaging planes on echocardiogram, respectively). In addi- 


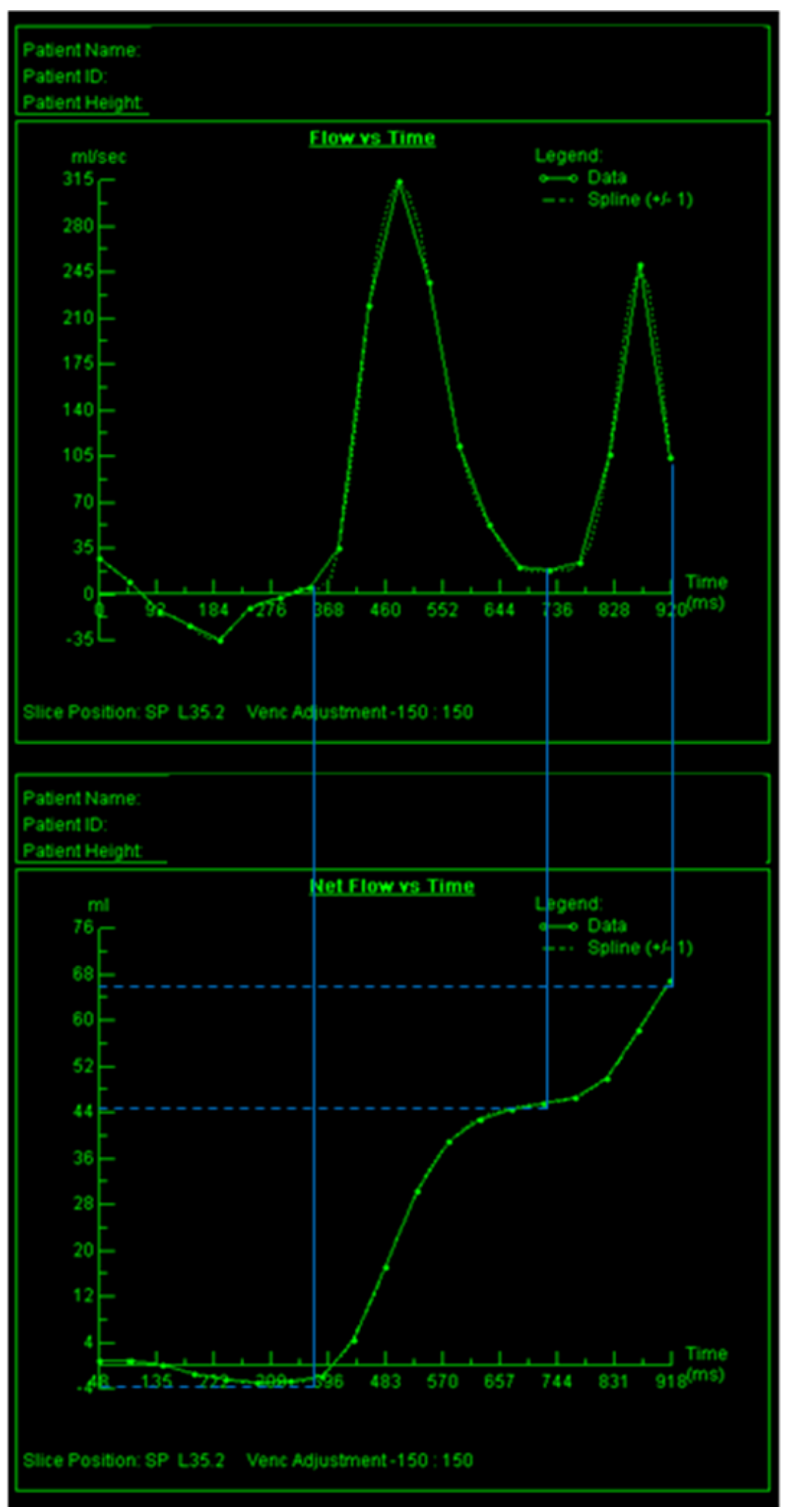

Figure 2. Determination of left atrial function from Ve-CMR flow data. The beginning and end times of the E-wave (passive ventricular filling) and A-wave (atrial contraction) were determined using the flow-time curve. Using these times, the inflow volume due to passive ventricular filling and atrial contraction was determined from the corresponding net flow-time curve. 
tion, height was measured in the three-chamber cine view (equivalent to the parasternal long-axis on echocardiogram). Left atrial volume was calculated by modeling the left atrium as a prolate ellipsoid:

$$
\text { Volume }=\frac{4 \pi}{3}\left(\frac{\mathrm{V}_{1}}{2}\right)\left(\frac{\mathrm{V}_{2}}{2}\right)\left(\frac{\mathrm{V}_{3}}{2}\right)
$$

where $\mathrm{V}_{1}=$ three-chamber view height, $\mathrm{V}_{2}=$ fourchamber view width and $\mathrm{V}_{3}=$ four-chamber view length [12].

\subsection{Statistical Analysis}

Continuous data are stated as mean $+/-$ standard deviation. Comparisons between groups were computed using 2-sample t-tests. Standard linear regression analysis was used to compare left atrial function and other cardiac parameters; all stated correlations are Pearson correlation coefficients. All statistical tests are two-tailed, with $\mathrm{P}<$ 0.05 considered statistically significant.

\section{RESULTS}

All patients had a complete CMR test. The mean age was $56+/-10$ years, the study group was $46 \%$ female, and mean body mass index was $28.5+/-5.2 \mathrm{~kg} / \mathrm{m}^{2}$. Fiftynine percent $(23 / 39)$ of the study group had a history of hypertension and approximately $40 \%$ (16/39) of the population had diabetes mellitus and left ventricular hypertrophy. Table 1 lists the baseline characteristics of the study group.

Mean left atrial function was $22.9 \%+/-14.5 \%$; the

Table 1. Baseline characteristics of study group.

\begin{tabular}{lc}
\hline Characteristic & Patient Group (n=39) \\
\hline Age (years) & $55.8+/-10.4$ \\
Females & $18(46 \%)$ \\
CAD Risk Factors & \\
Diabetes Mellitus & $16(41 \%)$ \\
Hypertension & $23(59 \%)$ \\
Cigarette Smoker & $6(15 \%)$ \\
Family History of CAD & $12(31 \%)$ \\
BMI & $28.5+/-5.2$ \\
Past Medical History & \\
History of Atrial Fibrillation & $4(10 \%)$ \\
Cardiomyopathy & $4(10 \%)$ \\
CHF & $4(10 \%)$ \\
Left Ventricular Hypertrophy & $16(41 \%)$ \\
Myocardial Infarction & $2(5 \%)$ \\
Stroke & $1(3 \%)$ \\
\hline
\end{tabular}

CAD: coronary artery disease, BMI: body mass index, CHF: congestive heart failure. range in left atrial function was $0 \%-57 \%$. Table 2 presents the mean values of several cardiac parameters and their correlation with left atrial function. The mean left ventricular ejection fraction was $58 \%+/-12 \%$; there was a significant positive correlation between atrial function and left ventricular ejection fraction $(\mathrm{r}=0.44, \mathrm{P}<0.01)$. The mean mitral regurgitation score was $1.1+/-1.0$; atrial function was inversely correlated with severity of mitral regurgitation $(\mathrm{r}=-0.60, \mathrm{P}<0.01)$. In addition, mean left atrial volume was $52.4+/-40.0 \mathrm{~cm}^{3}$; higher left atrial volume was associated with lower left atrial function $(\mathrm{r}=-0.36, \mathrm{P}=0.02)$. The mean $\mathrm{PR}$ interval was $167+/-30 \mathrm{~ms}$; no significant correlation was found between PR interval and left atrial function $(\mathrm{r}=0.03, \mathrm{P}=$ $0.87)$.

There were two patients with atrial fibrillation and one patient with complete heart block that were confirmed to have no atrial contraction and therefore zero left atrial function. Four additional patients had zero left atrial function despite having normal sinus rhythm on electrocardiogram and no history of arrhythmias. Of these four patients, none had severely reduced left ventricular ejection fraction. Three of these patients had diabetes, three had left ventricular hypertrophy, and two had history of hypertension. In addition, these patients had trivial or mild mitral regurgitation and normal PR intervals, except for one patient who had the longest PR interval $(231 \mathrm{~ms})$ of any patient in the study group. In addition, one patient reported a history of palpitations. The baseline characteristics and cardiac parameters of these four patients are listed in Table 3.

\section{DISCUSSION}

The results of this study are consistent with previously

Table 2. Cardiac parameters and correlation to left atrial function.

\begin{tabular}{lccc}
\hline Characteristic & Mean \pm SD & r & P-value \\
\hline Age (years) & $56 \pm 10$ & 0.17 & 0.30 \\
Inflow Volume $(\mathrm{mL})$ & $85 \pm 22$ & -0.05 & 0.78 \\
PR Interval (msec) & $167 \pm 30$ & 0.10 & 0.60 \\
Left Atrial Volume $\left(\mathrm{cm}^{3}\right)$ & $52 \pm 40$ & -0.36 & 0.02 \\
LV Wall Thickness: Anterior $(\mathrm{cm})$ & $0.9 \pm 0.3$ & -0.07 & 0.69 \\
LV Wall Thickness: Posterior $(\mathrm{cm})$ & $0.9 \pm 0.3$ & -0.25 & 0.12 \\
LV End Diastolic Diameter (cm) & $5.2 \pm 0.7$ & -0.26 & 0.11 \\
LV End Systolic Diameter (cm) & $3.6 \pm 0.9$ & -0.36 & 0.02 \\
LV Ejection Fraction-Visual (\%) & $58 \pm 12$ & 0.44 & $<0.01$ \\
Mitral Regurgitation Score & $1.1 \pm 1.0$ & -0.60 & $<0.01$ \\
\hline
\end{tabular}

LV: left ventricular, SD: standard deviation, R: correlation coefficient. 
Table 3. Patients with zero left atrial function with normal sinus rhythm and no history of arrhythmias.

\begin{tabular}{|c|c|c|c|c|}
\hline Patient & 1 & 2 & 3 & 4 \\
\hline Age (years) & 52 & 49 & 66 & 60 \\
\hline Females & M & M & $\mathrm{F}$ & M \\
\hline \multicolumn{5}{|l|}{ CAD Risk Factors } \\
\hline Diabetes Mellitus & $\mathrm{Y}$ & $\mathrm{Y}$ & $\mathrm{Y}$ & $\mathrm{N}$ \\
\hline Hypertension & $\mathrm{Y}$ & $\mathrm{N}$ & Y & $\mathrm{N}$ \\
\hline Cigarette Smoker & $\mathrm{Y}$ & $\mathrm{N}$ & $\mathrm{N}$ & $\mathrm{N}$ \\
\hline Family History of CAD & $\mathrm{N}$ & $\mathrm{N}$ & $\mathrm{N}$ & $\mathrm{Y}$ \\
\hline BMI & 33 & 32 & 27 & 30 \\
\hline \multicolumn{5}{|l|}{ Past Medical History } \\
\hline History of Atrial Fibrillation & $\mathrm{N}$ & $\mathrm{N}$ & $\mathrm{N}$ & $\mathrm{N}$ \\
\hline Cardiomyopathy & $\mathrm{N}$ & $\mathrm{N}$ & $\mathrm{N}$ & $\mathrm{N}$ \\
\hline $\mathrm{CHF}$ & $\mathrm{N}$ & $\mathrm{N}$ & $\mathrm{N}$ & $\mathrm{N}$ \\
\hline Left Ventricular Hypertrophy & Y & $\mathrm{Y}$ & Y & $\mathrm{N}$ \\
\hline Myocardial Infarction & $\mathrm{N}$ & $\mathrm{N}$ & $\mathrm{N}$ & $\mathrm{N}$ \\
\hline Stroke & $\mathrm{N}$ & $\mathrm{N}$ & $\mathrm{N}$ & $\mathrm{N}$ \\
\hline \multicolumn{5}{|l|}{ Cardiac Parameters } \\
\hline Inflow Volume (mL) & 73 & 86 & 62 & 92 \\
\hline PR Interval (msec) & 138 & 177 & 151 & 231 \\
\hline Left Atrial Volume $\left(\mathrm{cm}^{3}\right)$ & 31 & 52 & 51 & 31 \\
\hline LV Wall Thickness: Anterior (cm) & 1.1 & 1 & 0.7 & 0.9 \\
\hline LV Wall Thickness: Posterior (cm) & 1.2 & 1 & 0.7 & 0.7 \\
\hline LV End Diastolic Diameter (cm) & 5.8 & 6 & 4.8 & 5.7 \\
\hline LV End Systolic Diameter (cm) & 4.7 & 4.1 & 3.1 & 4.4 \\
\hline LV Ejection Fraction—Visual (\%) & $65 \%$ & $50 \%$ & $70 \%$ & $45 \%$ \\
\hline Mitral Regurgitation Score & 1 & 2 & 1 & 2 \\
\hline
\end{tabular}

CAD: coronary artery disease, $\mathrm{LV}$ : left ventricular.

reported results, including those using Ve-CMR, echocardiography, and invasive techniques (Table 4). However, our study group had a greater variability in atrial function than all of these prior studies. We postulate this to be due to the variety of clinical indications in our study group (referral population), compared to prior studies with either normal patients or patients with a specific pathology. Previous studies indicate a wide variability in left atrial function for patients with different disease states: for example, mean left atrial function in patients with severe mitral stenosis was found to be $9+/-$ $5 \%$, while mean left atrial function in patients with myocardial infarction to be $42+/-12 \%[13,14]$. Our study confirms that left atrial function can vary significantly within a clinical referral population.

Matsuda (1983) found an association between left ventricular ejection fraction and left atrial work (defined as atrial contraction volume); this correlation was attributed to the Frank-Starling law operating in the left atrium [14]. We postulate that the Frank-Starling law can explain the correlation between left atrial function and left ventricular ejection fraction found in this study: increased left atrial function leads to increased left ventricular enddiastolic volume, which would lead to increased left ventricular contractility and thus increased left ventricular ejection fraction.

The inverse correlation between left atrial volume and left atrial function may be due to increased passive left ventricular filling: increased left atrial volume would create an increased pressure gradient between the left atrium and left ventricle, which would lead to increased passive ventricular filling (E-wave), thereby decreasing the calculated left atrial function. Similarly, increased mitral regurgitation would lead to increased left atrial volume, thereby increasing passive ventricular filling and thus decreasing left atrial function; it has been shown that increased mitral regurgitation is correlated with increased peak E-wave velocity [15].

As detailed in the Results section, there were four patients that had zero left atrial function (absent A-wave) despite having normal sinus rhythm on ECG. Absent atrial function has been shown in patients with restrictive cardiomyopathy, such as amyloidosis [16], and pericardial constriction [17]. However, given that three of these patients had a history of LVH, we believe that a restricttive physiology (i.e. left ventricular hypertrophy leads to decreased left ventricular compliance, leading to impaired active filling) most likely explains these patients' zero left atrial function. Alternatively, it is possible that these patients may have had atrial fibrillation but recently spontaneously converted back into normal sinus rhythm, as there can be a delay of up to several weeks for recovery of mechanical function after normal electrical function has been restored [18]. One patient did have a history of palpitations and therefore possibly had undiagnosed paroxysmal atrial fibrillation. Finally, one patient's atrial function may be explained by possible E/A fusion from a markedly prolonged PR interval.

Limitations to this study include the lack of correction for mitral valve annulus motion, which may lead to calculation errors [19]. In addition, this study had a relatively small sample size. However, to our knowledge, this study is larger than any previous study using VeCMR to examine atrial contribution to left ventricular filling. By successfully evaluating left atrial function for 39 patients referred to CMR for a multitude of clinical indications, we have demonstrated that Ve-CMR can 
Table 4. Comparison of results with prior studies of left atrial function.

\begin{tabular}{|c|c|c|c|c|}
\hline Study & Method & $\mathrm{N}$ & Study Population & Atrial Function \\
\hline Bough et al. 1981 [5] & Radionuclide Angiography & 20 & Normal & $29.0 \%+/-10.0 \%$ \\
\hline Matsuda et al. 1983 [14] & Cineangiograms & 8 & Normal & $29.0 \%+/-10.0 \%$ \\
\hline Matsuda et al. 1986 [20] & Cineangiograms & 8 & Normal & $24.0 \%+/-7.0 \%$ \\
\hline Meisner et al. 1991 [13] & Doppler/2D Echocardiography & 11 & Normal & $25.0 \%+/-7.0 \%$ \\
\hline Hartiala et al. 1993 [8] & Ve-CMR & 10 & Normal & $25.9 \%+/-7.5 \%$ \\
\hline Hartiala et al. 1994 [9] & Ve-CMR & 12 & Normal & $24.9 \%+/-7.2 \%$ \\
\hline Oki et al. 1998 [21] & TEE & 30 & Normal & $39.6 \%+/-9.2 \%$ \\
\hline Prioli et al. 1998 [22] & Doppler/2D Echocardiography & 9 & Normal & $26.0 \%+/-3.0 \%$ \\
\hline Phan et al. 2009 [23] & Radionuclide Ventriculography & 15 & Normal & $33.0 \%+/-10.0 \%$ \\
\hline Matsuda et al. 1983 [14] & Cineangiograms & 10 & Myocardial infarction & $42.0 \%+/-12.0 \%$ \\
\hline Matsuda et al. 1986 [14] & Cineangiograms & 7 & Hypertensive heart disease & $35.0 \%+/-13.0 \%$ \\
\hline Meisner et al. 1991 [13] & Doppler/2D Echocardiography & 30 & Mitral stenosis & $18.0 \%+/-10.0 \%$ \\
\hline Hartiala et al. 1994 [9] & Ve-CMR & 9 & Aortic stenosis & $24.9 \%+/-7.2 \%$ \\
\hline Oki et al. 1998 [21] & TEE & 17 & Mild mitral stenosis & $17.9 \%+/-4.4 \%$ \\
\hline Oki et al. 1998 [21] & TEE & 19 & Moderate mitral stenosis & $13.4 \%+/-4.5 \%$ \\
\hline Prioli et al. 1998 [22] & Doppler/2D Echocardiography & 15 & Restrictive patterns & $19.0 \%+/-10.0 \%$ \\
\hline Prioli et al. 1998 [22] & Doppler/2D Echocardiography & 13 & Impaired relaxation & $38.0 \%+/-4.0 \%$ \\
\hline Phan et al. 2009 [23] & Radionuclide Ventriculography & 25 & $\mathrm{HF}$ w/preserved EF & $34.0 \%+/-12.0 \%$ \\
\hline Current Study & Ve-CMR & 39 & Referral & $22.9 \%+/-14.5 \%$ \\
\hline
\end{tabular}

Ve-CMR = velocity-encoded cardiac magnetic resonance: TEE = transesophageal echocardiography.

evaluate left atrial function on a wide variety of patients.

\section{CONCLUSION}

In this population of patients referred for a clinical CMR, there is a wide variability in left atrial function. Left atrial function is significantly associated with left ventricular ejection fraction, left atrial size, and mitral regurgitation.

\section{REFERENCES}

[1] Braunwald, E. and Frahm, C.J. (1961) Studies on Starling's law of the heart: IV. Observations on the hemodynamic functions of the left atrium in man. Circulation, 24, 633-642. doi:10.1161/01.CIR.24.3.633

[2] Thomas, J.D. and Weyman, A.E. (1991) Echocardiographic Doppler evaluation of left ventricular diastolic function. Circulation, 84, 977-990. doi:10.1161/01.CIR.84.3.977

[3] Gutman, J., Wang, Y.S., Wahr, D., et al. (1983) Normal left atrial function determined by 2-dimensional echocardiography. The American Journal of Cardiology, 51, 336-

\section{0. doi:10.1016/S0002-9149(83)80061-7}

[4] Rumberger, J.A., Weiss, R.M., Feiring, A.J., et al. (1989) Patterns of regional diastolic function in the normal human left ventricle: An ultrafast computed tomographic study. Journal of the American College of Cardiology, 14, 119-126. doi:10.1016/0735-1097(89)90061-2

[5] Bough, E.W., Gandsman, E.J. and Shulman, R.S. (1981) Measurement of normal left atrial function with gated radionuclide angiography. The American Journal of Cardiology, 48, 473-478. doi:10.1016/0002-9149(81)90075-8

[6] Jarvinen, V.M., Kupari, M.M., Poutanen, V.P., et al. (1996) A simplified method for the determination of left atrial size and function using cine magnetic resonance imaging. Magnetic Resonance Imaging, 14, 215-226. doi:10.1016/0730-725X(95)02098-E

[7] Mohiaddin, R.H., Amanuma, M., Kilner, P.J., et al. (1991) MR phase-shift velocity mapping of mitral and pulmonary venous flow. Journal of Computer Assisted Tomography, 15, 237-243. doi:10.1097/00004728-199103000-00009

[8] Hartiala, J.J., Mostbeck, G.H., Foster, E., et al. (1993) Velocity-encoded cine MRI in the evaluation of left ventricular diastolic function: Measurement of mitral valve and pulmonary vein flow velocities and flow volume 
across the mitral valve. American Heart Journal, 125, 1054-1066. doi:10.1016/0002-8703(93)90114-O

[9] Hartiala, J.J., Foster, E., Fujita, N., et al. (1994) Evaluation of left atrial contribution to left ventricular filling in aortic stenosis by velocity-encoded cine MRI. American Heart Journal, 127, 593-600. doi:10.1016/0002-8703(94)90668-8

[10] Mostbeck, G.H., Hartiala, J.J., Foster, E., et al. (1993) Right ventricular diastolic filling: Evaluation with velocity-encoded cine MRI. Journal of Computer Assisted Tomography, 17, 245-252. doi:10.1097/00004728-199303000-00013

[11] Heitner, J., Bhumireddy, G.P., Crowley, A.L., et al. (2012) Clinical application of cine-MRI in the visual assessment of mitral regurgitation compared to echocardiography and cardiac catheterization. PloS ONE, 7, e40491. doi:10.1371/journal.pone.0040491

[12] Sanfilippo, A.J., Abascal, V.M., Sheehan, M., et al. (1990) Atrial enlargement as a consequence of atrial fibrillation. A prospective echocardiographic study. Circulation, 82, 792-797. doi:10.1161/01.CIR.82.3.792

[13] Meisner, J.S., Keren, G., Pajaro, O.E., et al. (1991) Atrial contribution to ventricular filling in mitral stenosis. Circulation, 84, 1469-1480. doi:10.1161/01.CIR.84.4.1469

[14] Matsuda, Y., Toma, Y., Ogawa, H., et al. (1983) Importance of left atrial function in patients with myocardial infarction. Circulation, 67, 566-571. doi:10.1161/01.CIR.67.3.566

[15] Thomas, L., Foster, E. and Schiller, N.B. (1998) Peak mitral inflow velocity predicts mitral regurgitation severity. Journal of the American College of Cardiology, 31, 174-179. doi:10.1016/S0735-1097(97)00454-3

[16] Dubrey, S., Pollak, A., Skinner, M., et al. (1995) Atrial thrombi occurring during sinus rhythm in cardiac amyloidosis: Evidence for atrial electromechanical dissociation. British Heart Journal, 74, 541-544.

\section{doi:10.1136/hrt.74.5.541}

[17] Little, W.C. and Freeman, G.L. (2006) Pericardial disease. Circulation, 113, 1622-1632. doi:10.1161/CIRCULATIONAHA.105.561514

[18] Manning, W.J., Silverman, D.I., Katz, S.E., et al. (1994) Impaired left atrial mechanical function after cardioversion: Relation to the duration of atrial fibrillation. Journal of the American College of Cardiology, 23, 1535-1540. doi:10.1016/0735-1097(94)90652-1

[19] Westenberg, J.J., Roes, S.D., Ajmone Marsan, N., et al. (2008) Mitral valve and tricuspid valve blood flow: Accurate quantification with $3 \mathrm{D}$ velocity-encoded MR imaging with retrospective valve tracking. Radiology, 249, 792-800. doi:10.1148/radiol.2492080146

[20] Matsuda, Y., Toma, Y., Moritani, K., et al. (1986) Assessment of left atrial function in patients with hypertensive heart disease. Hypertension, 8, 779-785. doi:10.1161/01.HYP.8.9.779

[21] Oki, T., Iuchi, A., Tabata, T., et al. (1998) Left atrial contribution to left ventricular filling in patients with mitral stenosis: Combined analysis of transmitral and pulmonary venous flow velocities. Echocardiography, 15, 43-50. doi:10.1111/j.1540-8175.1998.tb00576.x

[22] Prioli, A., Marino, P., Lanzoni, L., et al. (1998) Increasing degrees of left ventricular filling impairment modulate left atrial function in humans. The American Journal of Cardiology, 82, 756-761. doi:10.1016/S0002-9149(98)00452-4

[23] Phan, T.T., Abozguia, K., Shivu, G.N., et al. (2009) Increased atrial contribution to left ventricular filling compensates for impaired early filling during exercise in heart failure with preserved ejection fraction. Journal of Cardiac Failure, 15, 890-897. doi:10.1016/j.cardfail.2009.06.440 